\title{
Low Bone Mass Is a Risk Factor in Periodontal Disease-Related Tooth Loss in Patients with Intellectual Disability
}

\author{
Yoko Numoto $^{1}$, Takayuki Mori ${ }^{2}$, Shigeru Maeda ${ }^{3}$, Yumiko Tomoyasu ${ }^{1}$, Hitoshi Higuchi ${ }^{3}$, Masahiko \\ Egusa $^{2}$ and Takuya Miyawaki ${ }^{1, *}$
}

${ }^{1}$ Department of Dental Anesthesiology and Special Care Dentistry, Okayama University, Graduate School of Medicine, Dentistry and Pharmaceutical Sciences, Okayama, Japan

${ }^{2}$ Center of the Special Needs Dentistry Okayama University Hospital, Okayama, Japan

${ }^{3}$ Department of Dental Anesthesiology, Okayama University Hospital, Okayama, Japan

\begin{abstract}
Teeth are fundamental to maintaining good quality of life, but are often lost prematurely in individuals with intellectual disability. Furthermore, since bone mass decreases in menopausal women, women with intellectual disability have an augmented risk of losing their teeth. However, the relationship between periodontal disease-related tooth loss and bone mass has never been studied specifically in patients with intellectual disability. This study evaluated this relationship in a retrospective cohort study.

Participants were female dental patients aged between 20 and 50 years and with an intellectual disability, who were treated in the Special Needs Dentistry unit of the Okayama University Hospital from January 2009 to March 2010. Logistic regression analysis was used to analyze which factors affect periodontal disease-related tooth loss. Information relating to 12 predictor variables, including age and bone mass level, was derived from medical records.

The 27 subjects had a total of 704 teeth at the time of initial examination, but 20 teeth $(2.8 \%)$ had been lost owing to periodontal disease by the time bone mass measurements were recorded. Results of the multinomial logistic regression analysis indicated a significant odds ratio for three items: number of missing teeth the time of initial examination, bone mass, and living environment.

This result suggests that low bone mass is an independent risk factor in tooth loss secondary to periodontal disease in patients with intellectual disability. Dentists should thus take account of this heightened risk of tooth loss when caring for post-menopausal women with intellectual disability.
\end{abstract}

Keywords: Bone mass, cohort study, intellectual disability, menopause, osteoporosis, periodontal disease.

\section{INTRODUCTION}

Teeth are fundamental to quality of life (QoL) throughout human life [1-4]. For elderly people and individuals with a disability, consumption of favorite foods is a major source of pleasure, since their disability tends to limit other enjoyments. However, individuals with disabilities have trouble with self-management and often find dental treatment intolerable, so maintenance of their oral health is more difficult than in non-disabled individuals. To compound the problem of premature tooth loss, these patients often have difficulty accepting dentures and other devices aimed at restoring a functional occlusion, so preventing tooth loss is becoming increasingly important in maintaining their QoL [5]. The number of teeth remaining has been correlated with chewing ability, nutritional intake, and general health status [6-8].

*Address correspondence to this author at the Department of Dental Anaesthesiology and Special Care Dentistry, Okayama University, Graduate School of Medicine, Dentistry and Pharmaceutical Sciences, 2-5-1 Shikatacho, Kita-ku, Okayama 700-8525, Japan; Tel/fax: +81-86-235-6721;

E-mail:miyawaki@md.okayama-u.ac.jp
However, the number of surviving teeth is considerably lower in people with intellectual disability (ID) than in those without ID [9]. Furthermore, individuals with Down syndrome (a representative chromosomal abnormality with associated ID) have an increased risk of periodontitis and reduced oral function [10]. Thus, individuals with ID have an increased risk of premature tooth loss and an associated reduction in their QoL.

Individuals with osteoporosis also experience an increased risk of losing teeth $[11,12]$. The progression of osteoporosis to the point of vertebral or femoral fracture can be devastating to QoL. Menopause, shortage of exercise, and malnutrition are key risk factors in developing osteoporosis [13]. Notably, individuals with severe ID generally take less exercise than normal, and are frequently malnourished owing to difficulty feeding (e.g. dysphagia). People with ID thus have a higher risk of osteoporosis [14-17]. Furthermore, young adults with Down syndrome have reduced bone mass [18]. Finally, the prevalence of epilepsy was significantly higher in autistic subjects with ID than in those without ID [19], and antiepileptic medicine is reportedly related to a decrease in bone mass [20]. Thus, patients with ID have mul- 
tiple factors that can predispose them to tooth loss. However, the relationship between bone mass and periodontal diseaserelated tooth loss specifically in patients with ID has not yet been studied. Therefore, we aimed to address this issue in this retrospective cohort study.

\section{MATERIALS AND METHODOLOGY}

\section{Study Design/Participants}

The investigators designed and implemented a retrospective cohort study. Participants were female dental patients, aged between 20 and 50 years, who were treated in the Special Needs Dentistry unit of Okayama University Hospital from January 2009 to March 2010. All participants had some congenital ID, and many also had autism, cerebral palsy, and/or other disabilities. Patients that could not tolerate measurements of bone mass level were not enrolled.

This study was approved by the Ethics Committee of the Graduate School of Medicine, Dentistry and Pharmaceutical Sciences at Okayama University (approval no. 579).

\section{Variables}

A logistic regression analysis was used to analyze the factors that affect periodontal disease, with odds ratios used to compare the degree of influence of each factor. The outcome variable of the logistic regression analysis was the number of periodontal disease-related tooth loss in the time between the initial examination and the bone mass measurement. "Periodontal disease-related teeth loss" was applicable when tooth mobility was classified as Grade III or above, or when the degree of alveolar bone resorption was more than half of the total root length (severe periodontitis).

A further 12 'explanatory variables' were also incorporated, including age (nominal variable: 35 years and older or less than 34 years), body mass index (BMI) (nominal variable: 25 and above or below 25), bone mass (nominal variable: normal or low), the number of missing teeth at the time of initial examination (continuous variable), number of years elapsed from initial examination (continuous variable), use of antiepileptic drugs (nominal variable: yes or no), smoking history (nominal variable: yes or no), diabetes mellitus (nominal variable: yes or no), living environment (nominal variable: own house or institution), periodic dental consultation (nominal variable: yes or no), tooth brushing (nominal variable: assisted or unassisted), and percentage bleeding on probing (BOP) (continuous variable). BOP was shown as percentage of number of bleeding points in that of all probing points in each participant. The third molars were not included in the aggregation and analysis of teeth in this study.

A unary logistic regression analysis was conducted for each variable. Next, we built a logistic model based on a variable increment method from the results of the unary logistic regression analysis based on the fidelity of the analysis model (AIC, likelihood ratio test), and performed a multinomial logistic regression analysis. We calculated the odds ratio and 95\% confidence interval from the multinomial logistic analysis and from these identified any significant differences. Microsoft Excel 2007 (Microsoft Japan, Tokyo, Japan) was used for data collection and Excel Statistic 2010 (SSRI, Tokyo, Japan) and STAT-VIEW 5.0 (SAS Institute
Japan, Tokyo, Japan) were used for statistical analysis. A significance level of $5 \%$ was set for all tests.

\section{Bone Mass Measurement}

A Benus $\alpha^{\circledR}$ ultrasound device (Nihon Kohden Co., Tokyo, Japan) was used to measure calcaneal bone mass. The trabecular bone area ratio was adopted as a parameter for bone mass. After cleaning the side of the heel and applying sufficient gel to facilitate measurement without interfering with the passage of the ultrasound waves, measurements were conducted with the subject's heel secured and with the device in a fixed position. Measurements were conducted at an appropriate temperature $\left(27.5-28.5^{\circ} \mathrm{C}\right)$. Patients were required to remain still for approximately $20 \mathrm{~s}$, therefore subjects whose ID precluded them from understanding the purpose of the study were unable to participate. To minimize body movement in these subjects, we employed behavioral adjustments including modeling methods and systemic desensitization; resistance to measurement was reduced as much as possible. Measurements were conducted twice and mean values of bone mass and the trabecular bone area ratio were recorded for each subject.

\section{RESULTS}

The 27 subjects (mean age \pm SD: $35.1 \pm 8.9$ years) had a cumulative total of 704 teeth at the time of initial examination, and $20(2.8 \%)$ were lost as periodontal disease-related tooth loss by the time bone mass measurements were taken. Probing depths of the 20 teeth lost to periodontal disease were more than $5 \mathrm{~mm}$, and mobility of teeth lost to periodontal disease was classified as Grade III in 11 teeth, Grade II in 8 teeth and Grade I in 1 tooth, whose pocket depth was 8 $\mathrm{mm}$.

Of the 12 explanatory variables, smoking history, diabetes mellitus, and periodic dental consultation were removed since all participants were categorized as 'no' for both smoking history and diabetes mellitus and 'yes' for periodic dental consultation. The remaining nine items were used as predictor variables.

Results of the unary logistic regression analysis were shown in Table 1. The number of missing teeth at the time of initial examination was 52. According to the measurement of bone mass, participants were divided into a normal bone mass group and a low bone mass group, consisted of 16 individuals with total 418 teeth and 11 individuals with total 286 teeth, respectively. Anti-epileptic drugs were used in 8 subjects with 208 teeth, and not used in 9 subjects with 496 teeth. BMI was more than 25 in 10 subjects with 266 teeth, and was less than 25 in 17 subject with 438 teeth. Sixteen subjects were no less than 35 years old, and their total number of teeth was 410 , while 11 subjects were less than 35 years old, and their total number of teeth was 294. Number of years elapsed was $10.1 \pm 7.9$ (mean $\pm \mathrm{SD})$. Nineteen subjects live in institutes, and their total number of teeth was 158 , while 10 subjects lives at their home, and their total number of teeth was 544. Tooth brushing was assisted in 22 subjects and their total number of teeth was 583 , while only 5 subjects brush their teeth by themselves, and their number of teeth was 119 . BOP $\%$ was $50.7 \pm 39.2($ mean $\pm \mathrm{SD})$. 
Table 1. Factors Affecting Periodontitis-Related Tooth Extraction in ID patients -Unary Logistic Regression

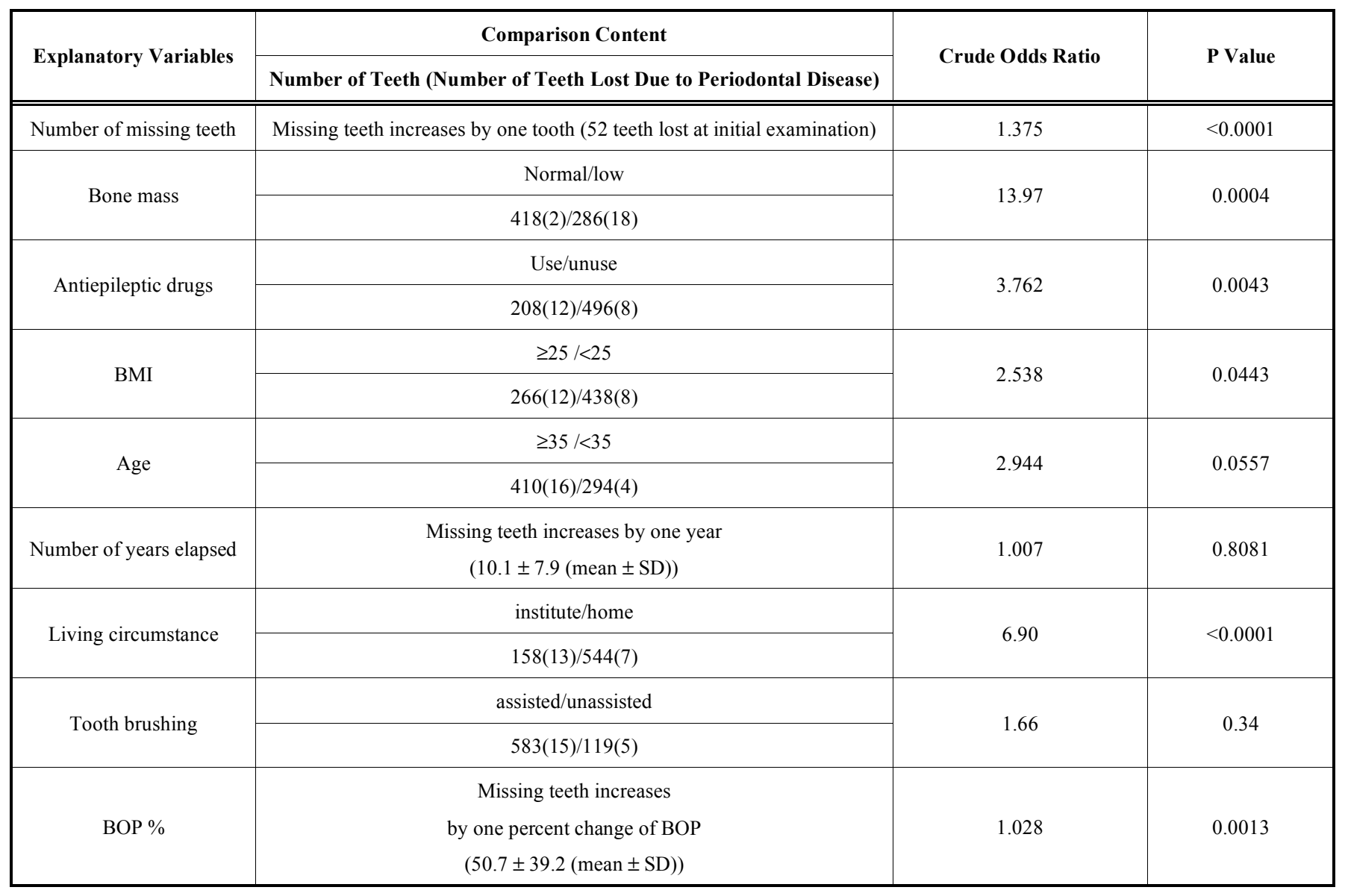

Table 2. Multinomial Logistics Model Analysis

\begin{tabular}{|c|c|c|c|}
\hline Explanatory Variables & Odds Ratio & $\mathbf{9 5 \%}$ Confidence Interval & P Value \\
\hline \hline Number of missing teeth & 1.54 & $1.24-1.84$ & $<0.0001$ \\
\hline Bone mass & 10.72 & $1.76-65.22$ & 0.01 \\
\hline Living circumstance & 5.34 & $1.83-15.54$ & 0.0021 \\
\hline BOP $\%$ & 1.04 & $0.997-1.032$ & 0.105 \\
\hline
\end{tabular}

Unary logistic regression analysis indicated that a significant correlation was seen between periodontitis-related tooth extraction and 1) missing teeth at the time of initial examination, 2) bone mass, 3) regular use of antiepileptic drugs, 4) BMI, 5) living arrangements, and 6) BOP \% (Table 1). The model that was most effective in the likelihood ratio test was a four-variable model of 1) number of missing teeth, 2) bone mass, 3) living arrangements, and 4) \% BOP. A multinomial logistic regression analysis was performed for these four items. Models with five or more variables were all deemed invalid based on the likelihood ratio tests.

Results of the multinomial logistic regression analysis indicated that the number of missing teeth at the time of initial examination, bone mass, and patient's living arrangements each had a significant odds ratio with regard to periodontal disease-related tooth loss (Table 2). Numerous missing teeth at the time of initial examination, low bone mass, and institutionalization all had notably high odds ratios $(1.54,10.72$, and 5.34 , respectively).

\section{DISCUSSION}

Low bone mass was identified as an independent predictor of periodontal disease-related tooth loss in this study of women with ID. In accordance with a previous report [16], our preliminary measurements confirmed that patients with ID have lower bone mass than those with no ID (data not shown). Thus, osteoporosis is considered a greater problem for individuals with ID than for those without. This finding should be incorporated in planning for treatment of periodontal disease in patients with ID, as prevention of periodontal disease by limiting osteoporosis-related bone loss would have significant benefits with regard to retaining oral function and maintaining QoL. 
The number of missing teeth at the time of initial examination was found to be a significant predictor of periodontal disease-related tooth loss. Patients with ID can often not tolerate dentures, meaning that their occlusal function depends entirely on their remaining teeth. The increased demand on this diminishing number of teeth often hastens the demise of these teeth and results in progression of the underlying periodontal disease. Institutionalization of patients with ID was also shown to dramatically affect the loss of teeth due to periodontal disease. Although the most basic treatment and preventative activity against periodontal disease is tooth brushing [21], most patients at our clinic require assistance in this regard because of their ID. This assistance is less readily available in an institution, where caregivers have responsibility for multiple patients; patients with ID fare better when living at home where they are more likely to receive undivided attention. This inadequacy of tooth brushing in the institutional environment likely underpins the progression of periodontal disease in these patients and thus their increased susceptibility to tooth loss.

The main outcome measured in this study was the number of teeth lost to periodontal disease. Although bacterial infection is the predominant direct cause of periodontal disease, various other factors have been purported as high-risk variables, including age, smoking history, diabetes mellitus, BOP, genetic factors, daily medication regimen, family environment, clinician experience, and number of missing teeth $[22,23]$. Genetic factors could not be assessed here because of difficulty in checking the genetic background of patients and their parents in this study. We selected a further 12 explanatory variables (age, BMI, bone mass, number of missing teeth at time of initial examination, years elapsed since initial examination, use of antiepileptic drugs, smoking history, diabetes mellitus, living arrangements, periodic dental consultation, tooth brushing, and BOP \%), of which three (smoking history, diabetes mellitus, and periodic dental consultation) were not analyzed because no variation was observed in these items. The 27 participants in this study had a cumulative total of 704 teeth; a prospective study design and larger sample size would be recommended for any further cohort study.

\section{CONCLUSION}

We conclude that the number of missing teeth at the time of initial examination, bone mass, and living arrangements are all significant independent risk factors in periodontal disease related tooth loss in intellectually disabled female subjects. This is the first article to reveal a relationship between bone mass and periodontal disease specifically in patients with ID. It is well known that these patients have increased susceptibility to dental disease and, owing to medical advances, are now living longer than previously expected [24]. Dentists should thus pay careful attention to managing the oral condition of menopausal women with ID, especially those with low bone mass, because they are suggested to represent a particularly high-risk group that are extremely vulnerable to tooth loss, which will have a consequent negative impact on their QOL.

\section{CONFLICT OF INTEREST}

The authors confirm that this article content has no conflicts of interest.

\section{ACKNOWLEDGEMENTS}

This work was supported in part by a Grant-in-Aid for Scientific Research (C): (24593152), from the Japan Society for the Promotion of Science.

\section{REFERENCES}

[1] Marino R, Albala C, Sanchez H, Cea X, Fuentes A. Self-assessed oral-health status and quality of life of older Chilean. Arch Gerontol Geriatr 2013;56:513-7.

[2] Sogur E, Onem E, Kalfa M, et al. Oral health and oral quality of life in inactive patients with familial Mediterranean fever without amyloidosis. Clin Exp Rheumatol 2013; 31(3 Suppl 77): 15-9.

[3] Niesten D, van Mourik K, van der Sanden W. The impact of having natural teeth on the QoL of frail dentulous older people: a qualitative study. BMC Public Health 2012; 12: 1839.

[4] Fontanive V, Abegg C, Tsakos G, Oliveira M. The association between clinical oral health and general quality of life: a population-based study of individuals aged 50-74 in Southern Brazil. Commun Dent Oral Epidemiol 2012; 41: 154-62.

[5] Hennequin M, Faulks D, Roux D. Accuracy of estimation of dental treatment need in special care patients. J Dent 2000; 28: 131-6.

[6] Kimura Y, Ogawa H, Yoshihara A, et al. Evaluation of chewing ability and its relationship with activities of daily living, depression, cognitive status and food intake in the communitydwelling elderly. Geriatr Gerontol Int 2013; 3(3): 718-25.

[7] Patel MH, Kumar JV, Moss ME. Diabetes and tooth loss: an analysis of data from the National Health and Nutrition Examination Survey, 2003-2004. J Am Dent Assoc 2013; 144: 478-85.

[8] Suzuki K, Nomura T, Sakurai M, Sugihara N, Yamanaka S, Matsukubo T. Relationship between number of present teeth and nutritional intake in institutionalized elderly. Bull Tokyo Dent Coll 2005; 46: 135-43.

[9] Gabre P, Martinsson T, Gahnberg L. Longitudinal study of dental caries, tooth mortality and interproximal bone loss in adults with intellectual disability. Eur J Oral Sci 2001; 109: 20-6.

[10] Hennequin M, Faulks D, Veyrune JL, Bourdiol P. Significance of oral health in persons with Down syndrome: a literature review. Dev Med Child Neurol 1999; 41: 275-83.

[11] Passos JS, Vianna MI, Gomes-Filho IS, et al. Osteoporosis/osteopenia as an independent factor associated with periodontitis in postmenopausal women: a case-control study. Osteoporos Int 2013; 24: 1275-83.

[12] Anil S, Preethanath RS, Almoharib HS, Kamath KP, Anand PS. Impact of Osteoporosis and Its Treatment on Oral Health. Am J Med Sci 2013, in press.

[13] lindsay R, Cosman F. Osteoporosis, In: Fauci AS, Braunwald E, Kasper DL, et al., Eds. Harrison's Principles of Internal Medicine. 17th ed. Columbus, McGraw-Hill 2008; pp. 2397-408.

[14] Wagemans AM, Fiolet JF, van der Linden ES, Menheere PP. Osteoporosis and intellectual disability: is there any relation? J Intellect Disabil Res 1998; 42 (Pt 5): 370-4.

[15] Petrone LR. Osteoporosis in adults with intellectual disabilities. South Med J 2012; 105: 87-92.

[16] Srikanth R, Cassidy G, Joiner C, Teeluckdharry S. Osteoporosis in people with intellectual disabilities: a review and a brief study of risk factors for osteoporosis in a community sample of people with intellectual disabilities. J Intellect Disabil Res 2010; 55: 53-62.

[17] Zylstra RG, Porter LL, Shapiro JL, Prater CD. Prevalence of osteoporosis in community-dwelling individuals with intellectual and/or developmental disabilities. J Am Med Dir Assoc 2008; 9: 109-13.

[18] Guijarro M, Valero C, Paule B, Gonzalez-Macias J, Riancho JA. Bone mass in young adults with Down syndrome. J Intellect Disabil Res 2008; 52: 182-9.

[19] Amiet C, Gourfinkel-An I, Bouzamondo A, et al. Epilepsy in autism is associated with intellectual disability and gender: evidence from a meta-analysis. Biol Psychiatry 2008; 64: 577-82. 
[20] Takahashi A, Onodera K, Kamei J, et al. Effects of chronic administration of zonisamide, an antiepileptic drug, on bone mineral density and their prevention with alfacalcidol in growing rats. J Pharmacol Sci 2003; 91: 313-8.

[21] Deacon SA, Glenny AM, Deery C, et al. Different powered toothbrushes for plaque control and gingival health. Cochrane Database Syst Rev 2010: CD004971.
[22] Kye W, Davidson R, Martin J, Engebretson S. Current status of periodontal risk assessment. J Evid Based Dent Pract 2012; 12: 211.

[23] Corbet EF. Oral diagnosis and treatment planning: part 3. Periodontal disease and assessment of risk. Br Dent J 2012; 213 111-21.

[24] Patja K, Iivanainen M, Vesala H, Oksanen H, Ruoppila I. Life expectancy of people with intellectual disability: a 35-year followup study. J Intellect Disabil Res 2000; 44 (Pt 5): 591-9.

Received: September 16, 2013

(C) Numoto et al.; Licensee Bentham Open.

This is an open access article licensed under the terms of the Creative Commons Attribution Non-Commercial License (http://creativecommons.org/licenses/by-nc/3.0/) which permits unrestricted, non-commercial use, distribution and reproduction in any medium, provided the work is properly cited. 